Sir,

\section{Postoperative infection and subtenon anaesthesia}

I read with interest the case report by Dahlmann et $a l^{1}$ describing a case of postoperative orbital cellulitis with a possible underlying infective cause from routine subtenon anaesthesia.

Similar case reports have been published earlier ${ }^{2}$ and more recently, ${ }^{3}$ and thus the comment by Dahlmann et al on being the first report of such case is erroneous. In our reported case, ${ }^{2}$ the signs of infection were also present very early on the second day after surgery, but the degree of orbital cellulitis and endophthalmitis progressed rapidly with poor visual outcome despite prompt systemic and topical treatment.

It was also unclear in the report by Dahlmann et al whether the patient had indeed received a preparation of the lids and conjunctiva with povidone-iodine before the subtenon injection. This procedure is now widely adopted by anaesthetists in the anaesthetic room together with appropriate draping, and has been shown to reduce the incidence of postoperative infection. ${ }^{4}$ Additional topical povidone-iodine applied into the conjunctival sac preoperatively would be recommended but only with an appropriately diluted concentration to avoid ocular toxicity.

\section{References}

1 Dahlmann AH, Appaswamy S, Headon MP. Orbital cellulitis following sub-tenon's anaesthesia. Eye 2002; 16(2): 200-201.

2 Lip PL, Moutsou M, Hero M. A post-operative complication far worse than endophthalmitis: the co-existing of orbital cellulitis. Br J Ophthalmol 2001; 85(5): 631-632.

3 Redmill B, Sanmdy C, Rose GE. Orbital cellulitis following corneal gluing under sub-tenon's local anaesthesia. Eye 2001; 15: 554-556.

4 Speaker MG, Menikoff JA. Prophylaxis of endophthalmitis with topical povidone-iodine. Ophthalmology 1991; 98: $1769-1775$.

P-L Lip

The Birmingham and Midland Eye Centre, City Hospital, Dudley Road Birmingham B18 7QH, UK

Correspondence: PL Lip

Tel: +441215076808

E-mail: pllw@doctors.org.uk

Eye (2004) 18, 229. doi:10.1038/sj.eye.6700612

Sir,

Progressive glaucoma in patients with Alzheimer's disease

With reference to 'Progressive Glaucoma in Patients with Alzheimer's Disease' (Bayer and Ferrari ${ }^{1}$ ), is it not time for an evidence-based national protocol for visual field analysis in glaucoma? It used to be common clinical knowledge that:

1. disc changes precede visual field change in glaucoma;

2. pathologically cupped discs are quite compatible with normal visual fields;

3. true glaucomatous field defects are never associated with plumb normal discs.

Is it necessary to get caught up in the pseudo-science of statistically significant visual field progression via Pattern Standard Deviation in patients with Alzheimer's disease? Such changes at the optic nerve head as described would surely have made the point alone without resorting to a refined visual field programme, potentially full of error and certainly profligate when one considers visual field analysis throughout the beleagured health service. For comorbidity in glaucoma clinic patients, see Beck and Karseras. ${ }^{2}$

\section{References}

1 Bayer AU, Ferrari F. Severe progression of glaucomatous optic neuropathy in patients with Alzheimer's disease. Eye 2002; 16: 209-212.

2 Beck M, Karseras AG. Disc assessment and visual field analysis in a hospital glaucoma population. $\mathrm{Br} J$ Ophthalmol 1982; 66: 99-101.

\section{AG Karseras}

27 Penlline, Witchruch

Cardiff CF14 2AA, UK

Correspondence: AG Karseras

Tel: +44 2920626665

E-mail: pakarseras@aol.com

Eye (2004) 18, 229. doi:10.1038/sj.eye.6700628 